\title{
Scientists' perspectives on consent in the context of biobanking research
}

\author{
Zubin Master ${ }^{\star, 1,2}$, Lisa Campo-Engelstein ${ }^{3}$ and Timothy Caulfield ${ }^{4}$
}

Most bioethics studies have focused on capturing the views of patients and the general public on research ethics issues related to informed consent for biobanking and only a handful of studies have examined the perceptions of scientists. Capturing the opinions of scientists is important because they are intimately involved with biobanks as collectors and users of samples and health information. In this study, we performed interviews with scientists followed by qualitative analysis to capture the diversity of perspectives on informed consent. We found that the majority of scientists in our study reported their preference for a general consent approach although they do not believe there to be a consensus on consent type. Despite their overall desire for a general consent model, many reported several concerns including donors needing some form of assurance that nothing unethical will be done with their samples and information. Finally, scientists reported mixed opinions about incorporating exclusion clauses in informed consent as a means of limiting some types of contentious research as a mechanism to assure donors that their samples and information are being handled appropriately. This study is one of the first to capture the views of scientists on informed consent in biobanking. Future studies should attempt to generalize findings on the perspectives of different scientists on informed consent for biobanking.

European Journal of Human Genetics (2015) 23, 569-574; doi:10.1038/ejhg.2014.143; published online 30 July 2014

\section{INTRODUCTION}

Of the many contemporary topics in research ethics, few have received as much attention as understanding the ethical, legal, and social (ELS) issues related to biorepository research - and more specifically around informed consent. Biobanks have emerged as a dominant research platform that often draws together features from genomic and cohort research methodologies, among other approaches. Many countries have invested substantially in state- or national-level biobanks. By collecting and storing biological specimens of numerous individuals along with their health, lifestyle and demographic information, biorepositories permit future research studies as new biomarkers are discovered. Biobanking research involves multiple players with different interests, including donors, those related to the donor, biobankers (scientists involved in the collection and storage of samples and information), the end users of biobanked material (ie, private or publically funded researchers and others working in institutions including law enforcement or government), and, possibly clinicians who must interpret findings. Despite the worldwide growth of biobanks, there are many unresolved research ethics challenges, including those associated with participant withdrawal, ${ }^{1}$ ownership and control of samples and information, ${ }^{2,3}$ commercialization, ${ }^{4}$ and the return of results and incidental findings. ${ }^{5}$ Yet none have received as much ethics discourse and policy debate than informed consent. ${ }^{6}$

Traditional ethics norms and practices require informed consent to be obtained before each new study. But in the case of biobanking research, a re-consent approach threatens the operation of large-scale biorepositories due to the added labor and associated costs needed to obtain consent for every new study from potentially thousands of participants. Due to these practical challenges, many biobanks adopt a general or broad consent approach where participants are asked to consent to all or a range of future research activities, ${ }^{6,7}$ (Table 1). However, general consent can be seen as limiting participant autonomy because donors do not have to re-consent for each study. It has also been suggested that this approach may not conform to existing legal norms. ${ }^{8}$ Several alternative, middle of the road models have been proposed that seek participant autonomy and interests to a greater degree than general consent while, at the same time, address particular issues associated with a full re-consent process (Table 1). ${ }^{9-13}$

The ethical and legal appropriateness of different consent models is one of the most divisive topics with the absence of a consensus among academic scholars, ${ }^{6}$ research policies, ${ }^{14}$ and public opinion data. ${ }^{15}$ While the bulk of research studies focus on capturing the perceptions of the general public and patients on research ethics issues related to biobanking, ${ }^{16-18}$ only a few have focused on understanding the views of scientists who may manage biobanks ${ }^{19-25}$ and even fewer concentrate on scientists' perceptions of informed consent. ${ }^{26,27}$ It is important to capture the opinions of scientists as they run biobanks and are the end users of biorepository samples and information and thus have first-hand knowledge on biobank functions. In this paper, we report the results of a pilot study that involved interviewing scientists who attended the 2013 European Lab Automation conference in Hamburg, Germany. Our results add to the ethical discourse on stakeholder perception when compared with other empirical data on the opinions of patients and the general public.

\section{MATERIALS AND METHODS}

We used a qualitative research approach to understand the views of scientists who attended the European Lab Automation (ELA) 2013 - Biobanking

${ }^{1}$ Alden March Bioethics Institute, Albany Medical College, Albany, NY, USA; ${ }^{2}$ Health Law Institute, Law Centre, University of Alberta, Edmonton, Alberta, Canada; ${ }^{3}$ Alden March Bioethics Institute, OBGYN Department, Albany Medical College, Albany, NY, USA; ${ }^{4}$ Health Law Institute, Faculty of Law and School of Public Health, Law Centre, University of Alberta, Edmonton, Alberta, Canada

${ }^{*}$ Correspondence: Dr Z Master, Alden March Bioethics Institute, Albany Medical College, 47 New Scotland Avenue, MC 153, Albany, NY 12208-3478, USA. Tel: +1 518262 1548; Fax: +1 518262 6856; E-mail: zubin@zubsplace.com

Received 1 February 2014; revised 28 May 2014; accepted 25 June 2014; published online 30 July 2014 
Table 1 Models of informed consent for biobanking

\begin{tabular}{|c|c|}
\hline Re-consent & Donors are informed and are required to consent to the current study and to each future research study involving the use of their samples and information \\
\hline Presumed & Consent is presumed to have been given by donors to use their samples and information for all research unless they actively choose to opt out \\
\hline Seneral & $\begin{array}{l}\text { Donors can actively consent once for the current study and all future research involving the general use of their samples and information. Other terms: } \\
\text { open, generic. and blanket consent }\end{array}$ \\
\hline Broad & $\begin{array}{l}\text { Donors can actively consent once for the current study and all future research within a broad field, eg, cancer, diabetes, or heart disease. Many peopl } \\
\text { view broad and blanket consent to be similar or, rightly or not, use the terms interchangeably }\end{array}$ \\
\hline Tiered & $\begin{array}{l}\text { Donors can actively consent once for the current study and choose one or more broad fields of research or other options, ie, whether they would be } \\
\text { willing to have their samples used in research that result in commercialization. Other terms: line item or multilayered consent }\end{array}$ \\
\hline Delegated trustee & Donors can transfer consent to a trustee who is at arms-distance length from the biobank and consents on behalf of donors \\
\hline $\begin{array}{l}\text { Third party } \\
\text { oversight }\end{array}$ & $\begin{array}{l}\text { Donors can actively consent to a general, broad or other model, but an ethics board must approve the study before the commencement of research us } \\
\text { stored samples and information. This approach is emerging as a common component of biobanking governance schemes }\end{array}$ \\
\hline
\end{tabular}

conference in Hamburg, Germany on 6-7 June 2013. The ELA conference catered almost exclusively to scientists who run or work with biobanks, collect and use samples from biobanks, or perform high-throughput genetics and metabolic studies among other types of research and thus served as a convenience sample for our study. A total of 15 scientists were interviewed from the following countries: Germany (7), United Kingdom (2), Netherlands (2), United States (2), Luxembourg (1), and Finland (1) and involved 4 women and 11 men. Participants were all scientists with education and training in medicine and/or the health or biological sciences. While occupying different positions, most scientists worked in research and development, or management within for-profit pharmaceutical or biotechnology companies (10), and a third worked in not-for-profit organizations including biobanks, laboratories, foundations, and charitable organizations (5). Participants covered a range of health research areas (ie, neuroscience, cancer, and cardiovascular disease) and employed cell and molecular biology, biochemistry, and cell biology techniques. Some scientists also had backgrounds in biophysics and chemistry.

On the basis of the previous literature examining ELS issues associated with biobanking, ${ }^{6,7,15,28}$ we developed an interview guide containing eight semistructured questions and six demographic questions (Supplementary Material). Two vignettes were used to explain different models of consent and exclusion clauses. A series of short interviews (10-20 min) were conducted in English by ZM at the Congress Center Hamburg. Permission to conduct the study was obtained from conference organizers and ethics approval was obtained from the Albany Medical Center Committee on Research Involving Human Subjects Institutional Review Board before the commencement of research. All participants received an information sheet and a verbal explanation of the study and gave informed consent.

Interviews were conducted until we observed repetition of several viewpoints (data saturation). All interviews were recorded using a digital voice recorder, transcribed, and transcripts were validated against audio recordings and de-identified of any personal information of participants. Analysis of transcripts was based on an abridged grounded theory approach ${ }^{29}$ with constant comparison analysis. ${ }^{30}$ A coding framework (codebook) was inductively developed by both coders (ZM and LCE) based upon the analysis of three transcripts known to have in-depth interviews. Both coders (ZM and LCE) independently coded all 15 transcripts for common themes using qualitative software QSR International NVivo version 10 (www.qsrinternational.com). First, a basal level of simple descriptive coding was performed followed by further in-depth comparison of codes. A consensus of the emergent themes and supporting quotes was obtained among both coders. A copy of the full basal coding of transcripts will be provided upon request to the corresponding author.

\section{RESULTS AND DISCUSSION}

Scientist do not perceive a consensus on informed consent

On the basis of a small number of national- or state-level biobanks ${ }^{6}$ and cancer biobanks ${ }^{7}$ throughout the world, two independent studies indicate that most biobanks surveyed operate using a broad or general consent approach. Without informing participants of these studies, we asked scientists whether they thought there was a consensus on the appropriateness of various forms of consent in the context of biobanks to get a sense of how they perceive research ethics policy debates. The majority of scientists reported that there is no consensus for a variety of reasons. Several scientists explained there are too many stakeholders at the table with differing opinions. As one scientist stated,

I guess because people ... just have completely different views on what is important and what is not. For me as a biobanker, it's primarily important that I can keep the biobank running and from that point of view, I can have only broad consent ... otherwise, just $[s i c]$ we would have to shut down. That is very simple. And I think others who just concentrate let's say only on ethical issues, for them this is not of primary concern. They may have [a] completely different view on that. (Participant 10)

Several others reported that different jurisdictions have different rules on privacy and consent that determine how biobanks operate. In other words, disparate national and state policies have led to a lack of consensus. One interviewee explained,

Well, I think it depends on where the biobank is located. So, there are different laws between different countries. (Participant 15)

Only one scientist had a dissenting opinion reporting that 'general consent' is the accepted norm because one cannot predict the future use of samples and that general consent is easier to explain to the public:

I thought that to be the $[s i c]$ general consent ... because it's for a non-expert or a patient. Also, it's very difficult to decide which type of research may be important or which will be important in the future for example. And I think it's easier to explain to a nonexpert in a ... more general way. (Participant 8)

These results suggest that while many legal and ethical scholars might believe there to be a consensus around the use of general or broad consent for biobanking, ${ }^{31-33}$ this sample of scientists expressed a different opinion. Many of our participants mentioned that their views were based on their experience with informed consent and from working with many stakeholders including patients/donors, patient advocacy groups, ethics boards and funders. While past studies, including our own work, have found that general or broad consent is common in large national or regional biobanks, ${ }^{6,7}$ diversity in the consent approach may increase when biobanks operate at smaller scales, including those within companies or university departments. This might partially explain why scientists interviewed in this study, a group that deals with a range of biobanks, believed there is no consensus in the consent approach for biobanking. 
Scientists prefer a general consent approach but understand potential ethical qualms raised by donors

While most scientists felt there was no consensus, the majority also expressed a preference for a general or broad consent approach. This position was often justified on grounds that this approach is important for the growth of science and by pointing to the practical difficulties of re-consent, including the fact that it is resource intensive, the possibility of losing valuable specimens, and the difficulty in predicting future research needs (Table 2).

Our findings are consistent with much of the relevant research on point. In a qualitative research project, Whitley et al ${ }^{27}$ reported that scientists noted the difficulty in predicting the future research uses of biological samples and information. Similarly, a study examining genomic researchers' attitudes on informed consent found that the majority preferred a general or broad consent approach. ${ }^{26}$ In contrast, Garrison and $\mathrm{Cho}^{25}$ found that there was no consensus on researcher preferences of general or broad over specific consent, although interview questions in their study were not specifically asking for such preferences.

While the majority desired a general or broad consent approach, two interviewees did mention their preference for tiered consent because this way, donors can retain some form of control over their samples and information as one interviewee explains,

If I have donated a sample and someone is going to ask me each time this sample is used, I need to understand what it's used for in detail to have a basis for my decision. And I think I wouldn't be able to understand each type of research that's being done. So, I think being able to limit it to certain areas would be completely enough for me as a donor to decide because it's more a basic ethical thing. So, I don't want my sample to be used for certain type of research projects or for certain type of grouping or so because I am concerned about my individual rights. (Participant 15)

In addition, a couple of scientists in our study explained that they used a third party consent approach where the ethics committee had to approve each new study, which reassures donors that their samples and information are being used for legitimate purposes. Not surprisingly, no scientists in our study preferred a re-consent approach. We observed similar results in a separate on-going study surveying Canadian scientists where $69.2 \%$ preferred a one-time general consent, $30.8 \%$ preferred a tiered consent approach, and none favored a re-consent approach (unpublished observations).

\section{Donors' need to know}

We asked scientists to state potential advantages and disadvantages of different consent models. One of the dominant themes was scientists' recognition that donors want to know how their sample and information will be used to ensure it aligns with their values and beliefs and that general consent may not provide sufficient information to allow this to happen. Despite scientists' preference for broad or general consent, they recognize that it may not be ideal, at least from a donor's perspective. Participants also noted several areas that might be viewed as contentious by donors, including mental health research, commercialization, and collaborations with industry, drug or cosmetic testing, military research, and cloning experiments (Table 3).

While many of the interviewees recognized that broad and general consent might be problematic from the perspective of patient autonomy, at least a few suggested that a re-consent process was not, necessarily, the answer. For example, one of the interviewees explained the difficulties of re-consenting and argued that it would be best to have the relevant information during initial consent as biobank participants will not want to be recontacted.

I don't think that the patient himself would want to re-consent specifically. They only want to know that nothing questionable is done with their specimen. Right? And they would want to do that with a primary consent. So re-consenting every time and again, I would like to do this and I would like to do that, or could we use your specimen for this purpose as well is not desirable [and] is logistically extremely complicated. And I don't think that a patient would want to be contacted two or three times totally independent of and whenever. It could be years after the first contact. And that's not gonna work because you are not gonna find the people anymore. (Participant 2)

Despite having a general or broad consent in her/his affiliated biobank, one scientist reported that informing patients thoroughly serves to increase participation because donors want the research to help themselves or others:

We have done a lot of work in [City X] ... and the sign up to [our]... cancer research trials is I think twice the national average. And that's primarily driven by very clued up research nurses, researchers, clinicians. It's the people that are getting involved and talking to the patients. And almost without exception, when patients are confronted with a well described problem and the reason that their sample can have a difference, patients... don't care what happens to their DNA, their blood, or their other piece of tumor. If it's gonna help them, fantastic... But if it's gonna help the next person, they may say, you know, 'I want to help out too'. (Participant 5)

This response indicates that a more involved and content-rich informed consent process might show respect to donors and can go a long way to increase participation and foster trust.

This idea that patients prefer broad consent is one often heard in the research community. ${ }^{34,35}$ And while it is true that several studies

\section{Table 2 Scientists' statements advocating for a general or broad consent approach}

\footnotetext{
Participant 2 Of course, the desired model would be any form which is as open as possible

Participant 10 I don't have any real concerns because I see it more of a thought that broad consent makes sense. Either you want to support science and health research or not. And why would you do one thing and not the other

Participant 12 Since it's being dragged out of my body, if it could bring some good to somebody somewhere sometime, even in the future, then good show

Participant 13 If I was in a position to donate my samples into a biobank, then I would rather try to keep it open to a general research because in the end, I hope to get feedback from that you know. If I am a patient, then I want to have treatment from different areas and I would not see this as an advantage to restrict it to only certain fields. That's why I cannot really understand why people want to restrict it to certain fields. So, if you tick it and have yes/no questions for a certain area, I think it would be rather a disadvantage if you restrict it to only a certain field
} 
have found that many in the general public and patient participants prefer general consent, this view is certainly not unanimous. Studies have found that many desire to have some form of ongoing control, such as a tiered or re-consent approach. ${ }^{15,16,18}$ Of particular relevance, one study showed that $67 \%$ of Europeans opted for specific (re-consent) whereas only $24 \%$ opted for a general (one-time) or presumed consent models. ${ }^{36}$ The authors explain the differences in participation and informed consent from different European nations to be due to the public's engagement with biobanks, views about privacy and data protection, and trust in the actors and institutions involved in biobanks.

In total, our results show that scientists prefer a general consent approach, but they are concerned and understand the issues donors might have with respect to having some form of continuing knowledge about the use of, and control over, their samples and information. And this desire for retaining control is arguably heightened in cases where the research or use of samples and information could be discriminatory or stigmatizing ${ }^{37-39}$ and when the research goals conflict with cultural or religious beliefs. ${ }^{40,41}$ Here, donor support and participation for biobanks might wane for stigmatizing or contentious areas of research and donors would be reluctant to have their samples be freely used and shared with others.

\section{Scientists have mixed views about excluding contentious areas of research and sharing samples and data}

Several public perception studies indicate that while most donors are willing to provide samples and information for research involving a range of diseases, they may be reluctant to freely provide biological material and information for areas of research that may be discriminatory or stigmatizing, for example, HIV research, schizophrenia research, race/ancestry determination, inbreeding, alcoholism, and sexual orientation just to name a few. ${ }^{37-41}$ Similarly, people may be disinclined to provide samples and information and have their data shared with organizations they deem less trustworthy including private industry, government and law enforcement agencies, employers, biotech companies, and insurance agencies, ${ }^{17,18,38,39}$ On the basis of this rationale, a conceptual proposal was developed that some biobanks, mostly smaller disease-specific biobanks, eg, a cancer biobank, may benefit from incorporating exclusion clauses in their informed consent process. ${ }^{28}$

Exclusion clauses are written within informed consent forms and can be explained verbally. Exclusion clauses omit areas of contentious research the biobankers do not foresee undertaking in the future and exclude organizations that biobankers do not anticipate sharing samples and information with and are perhaps deemed less trustworthy by the public. For example, a biobank specializing in leukemia research is unlikely to work on alcoholism or share data with insurance companies. This biobank may benefit from excluding several areas of contentious research and sharing. There are several advantages to using exclusion clauses for smaller or specialized biobanks: they are simple and easy to incorporate into all consent models including general, broad, and tiered consent; they raise awareness and educate donors about the different uses of banked samples and information; they increase transparency and accountability; and they offer assurances to donors that their samples will be handled with care. ${ }^{28}$

We wanted to know whether our participants thought exclusion clauses could work, what might be some of the advantages and disadvantages, and whether they would be willing to use exclusion clauses in informed consent. A majority of the scientists explained that exclusion clauses would work under certain conditions. Yet, scientists had mixed opinions when asked whether they would use them.

Many scientists reported several advantages and disadvantages of exclusion clauses. Some of the advantages enumerated were increasing transparency, educating donors, and comforting donors from knowing that their samples and information will be used appropriately (Table 4).

\section{Table 3 Scientists' statements explaining the desire of donors to have knowledge and control over their samples}

Participant 2 It would be concerning for the patient because the patient may be insecure about the use of the specimen and the level of information that's gonna be created with, out of that specimen... So, I think there is a way where we can find a good sound basis with our patients which provides them with the confidence that nothing weird is happening with their specimen. And then, they would let it go ... As I said earlier, the participant wants to make sure that nothing which is not within their ethical framework is going to happen with their specimen ... So, if an individual would definitely make sure that none of their material is being used for, I don't know, drug testing or for cosmetics testing or God knows what might [sic] unlikely scenarios

Participant 4 Yeah and like ... mental diseases or that kind of like syndromes. And maybe those kind[s] of studies where you need to also know the personal history of the people and you cannot use the results anonymously

Participant 7 Maybe that your material might be used for cloning experiments. I think that will be ... a major issue still. Yeah, as I said you don't know what it is going to be used for. So, I think that even though you have given a general consent, but [sic] you have to set your boundaries

Participant 15 So, it should be well, somehow protection of the individuals and if I donate a sample, I would like to be able to tell what the sample is used for. For example, I don't want my sample to be used for let's say, military purposes ... So for me as a donor, I want my sample to be used for some purpose that I consider is a good purpose

Table 4 Scientists' statements on the advantages of exclusion clauses in informed consent for biobanking

Participant 3 At least, you know for me, I am feeling like much more comfortable ... when giving consent ... Probably like I said you know, the advantage is that you know how and where it's going to be used and in many cases, people would be much more agreeable to that concept

Participant 13 I think it's quite transparent, the exclusion clause because you point people to certain fields that they themselves don't really know that the sample could be used for this kind of research. That's an advantage, but as I said before, you need to try to mention all possible fields of research and not just leaving some fields out

Participant 15 So, again from a donor's point of view, I think this would ... give me a good feeling if I know that at least there is some regulation behind the use of my samples. Well, so, talking from a researcher's point of view, I think this wouldn't do any harm because I am focused as a researcher and maybe if I want to change my area of research in the future, I think still I couldn't use these samples for this type of research or I would have to sign another agreement for that ... So, it makes people think about what they are doing with these samples and it gives a little bit more consciousness about [the] ethics topics that are linked with these biobanks 
While some scientists were confident that exclusions could be a helpful strategy, others were more skeptical. One scientist reported preferring a general consent approach, but would use exclusion clauses only if it were to increase recruitment. One of the disadvantages about exclusion clauses originally proposed was that they may decrease participant recruitment because it might cause donors to second guess what biobanks are doing when they exclude contentious research. ${ }^{28}$ However, a few scientists reported the opposite stating that exclusion clauses might actually increase donor participation:

I think when you have exclusion clauses in there, you could probably increase the amount of volunteers that donate their samples ... I mean we are all concerned about our data ... and if you get the confirmation of the company or of the person that you provide your data to that it will not be used for certain field $[\mathrm{s}]$ that you don't want it to be used for ... then the consent ... is to my opinion higher. (Participant 13)

These scientists seem to imply that exclusion clauses could increase recruitment because donors would have greater trust in biobanks knowing that biobanks were using their samples in ways that align with their beliefs.

For instance, one scientist who operates a biobank containing $\sim 500000$ samples used an exclusion clause in the general consent approach to omit any genetics research where DNA would be sequenced and further analysis performed. The biobanker reported that the exclusion clause gave donors an extra bit of assurance that their samples would be used in an ethical way and that some oversight was provided. While the researchers can perform any research using other biomarkers, the interviewee reasoned that the exclusion was incorporated due to ethical exceptionalism over genetics-based research. Similar results were also found in a study where research ethics committees in the United Kingdom did not readily allow general consent forms permitting any future research and some committees distinguished DNA-related tests from other forms of research. ${ }^{27}$ While it is interesting to see that exclusions are being incorporated into informed consent applications for biobanking, it remains to be seen whether they can be used to omit contentious research and sharing.

In addition to the advantages, many scientists reported a range of disadvantages including that exclusion clauses may provide a false impression to donors that the excluded areas are the only types of contentious research, that exclusion clauses may make participants question the type of research biobanks perform, and that biobankers would simply not know what to exclude or that they may exclude something irrelevant to the group(s) they are studying and instead serves to eliminate potentially beneficial research. One interviewee asserted that exclusion clauses could not work for any for-profit biobanks because any limits placed on research or sharing that would decrease profit would be contrary to the company's interests and there would be little rationale for them to use it. The disadvantages and the mixed opinions of scientists on whether exclusion clauses would be useful provide greater insight into whether they can be practically applied, even for small-scale biobanks. It is not surprising that some scientists have reservations about exclusion clauses as they limit fully open consent and restricts some areas of biobanking research. At the same time, however, scientists surveyed here also reported seeing value in helping assure patients that their samples and information will be used in an ethical manner. These findings also suggest that perhaps other stakeholders, including the general public, may differ in their opinions on the utility of exclusion clauses, a topic that deserves further investigation.

\section{Summary}

As biobanks continue to flourish, the ethical and legal appropriateness of different informed consent models needs to be determined. While the scientific community cannot, of course, dictate consent policy, ${ }^{8}$ scientists have an important role in the consent process and provide a valuable and necessary perspective that should help to inform consent policy. As collectors and users of biosamples and health information, they have unique knowledge on the inner workings of biobanks. In our pilot study, we found that while most scientists prefer a general or broad consent model, they do not perceive a consensus on informed consent. This is in contrast to some published studies demonstrating that most national- or state/provincial-level biobanks adopt a general or broad consent approach. Scientists in our study also understand and sympathize with donors knowing that they desire some form of control and want to know how their samples and information are being used, especially when the research is not aligned with participants' values. Finally, scientists interviewed here have mixed views about adopting exclusion clauses in informed consent procedures. While several see their benefits, others are more reluctant to incorporate them and would rather stick with a general consent approach. One of the limits of our study is that it is a pilot analysis of a small number of scientists. While this research has enriched our understanding of the views of scientists using a qualitative approach, future research should complement the work performed here and attempt to generalize findings by surveying a large cohort of scientists.

\section{CONFLICT OF INTEREST}

The authors declare no conflict of interest.

\section{ACKNOWLEDGEMENTS}

We are most appreciative for the scientists who graciously volunteered their time to participate in the interview. We would also like to thank the Select Bioscience meeting organizers, in particular Ms Hannah Tweed, for permitting us to conduct interviews at the ELA 2013 conference. ZM was invited to the ELA 2013 conference to speak on ELS issues of biobanking and registration and accommodations were covered by conference organizers. We would like to thank the two anonymous reviewers for thoughtful comments and Mr Michael S. Opare-Addo for technical assistance on the project. Transcription of interviews was performed by Cabbage Tree Solutions. This work was in part supported by Professor Caulfield's grants from the Cancer Stem Cell Consortium (CSCC) with funding from the Government of Canada through Genome Canada and the Ontario Genomics Institute (OGI-047); Allergy, Genes and Environment Network of Centres of Excellence Inc. (AllerGen); Paceomics (Genome Alberta); the Interdisciplinary Chronic Disease Collaboration funded by the Alberta Heritage Foundation for Medical Research (Alberta Innovates); and the cbcf Tumor Bank. We are most appreciative for project support by Ms Faria Grant, Ms Robyn Hyde-Lay and Ms Nicki Baron.

1 Elger B: Withdrawal of consent and destruction of samples; in Elger B, Biller-Andorno N, Mauron A, Capron AM (eds) Ethical Issues in Governing Biobanks. Global Perspectives. Farnham, Surrey, England: Ashgate Publishing Limited, 2008; Chapter 8, pp 131-165.

2 Charo RA: Body of research—ownership and use of human tissue. N Engl J Med 2006; 355: 1517-1519.

3 Boggio A: Ownership of samples and data and territorial restrictions concerning data and samples beyond national boundaries; in Elger B, Biller-Andorno N, Mauron A, Capron AM (eds) Ethical Issues in Governing Biobanks. Global Perspectives. Farnham, Surrey, England: Ashgate Publishing Limited, 2008; Chapter 11, pp 197-205.

4 Caulfield $T$, Burningham $S$, Joly $Y$ et al: A review of the key issues associated with the commercialization of biobanks. J Law Biosci 2014; 1: 94-110.

5 Wolf SM, Lawrenz FP, Nelson CA et al: Managing incidental findings in human subjects research: analysis and recommendations. J Law Med Ethics 2008; 36: 219-248.

6 Master Z, Nelson E, Murdoch B, Caulfield T: Biobanks, consent, and claims of consensus. Nat Methods 2012; 9: 885-888. 
7 Allen C, Foulkes WD: Qualitative thematic analysis of consent forms used in cancer genome sequencing. BMC Med Ethics 2011; 12: 14.

8 Caulfield T: Biobanks and blanket consent: the proper place of the public good and public perception rationales. King's Law J 2007; 18: 209-226.

9 Sharp RR, Foster MW: An analysis of research guidelines on the collection and use of human biological materials from American Indian and Alaskan Native communities. Jurimetrics 2002; 42: 165-186.

10 Winickoff DE, Winicoff RN: The charitable trust as a model for genomic biobanks. N Engl J Med 2003; 349: 1180-1184.

11 Thasler WE, Schlott T, Kalkuhl A et al: Human tissue for in vitro research as an alternative to animal experiments: a charitable "honest broker" model to fulfill ethical and legal regulations and to protect research participants. Altern Lab Anim 2006; 34 387-392.

12 Helft PR, Champion VL, Eckles R, Johnson CS, Meslin EM: Cancer patients' attitudes toward future research uses of stored human biological materials. J Empir Res Hum Res Ethics 2007; 2: 15-22.

13 Harmon SHE: Semantic, pedantic or paradigm shift? Recruitment, retention and property in modern population biobanking. Eur J Health Law 2009; 16: 27-43.

14 Salvaterra E, Lecchi L, Giovanelli S et al: Banking together. A unified model of informed consent for biobanking. EMBO Rep 2008; 9: 307-313.

15 Rachul C, McGuire A, Caulfield T: Public perceptions and biobanking: what does the research really say? Stud Ethics Law Technol 2012; 6: Article 3.

16 Murphy J, Scott J, Kaufman D, Geller G, LeRoy L, Hudson K: Public perspectives on informed consent for biobanking. Am J Public Health 2009; 99: 2128-2134.

17 Caulfield T, Rachul C, Nelson E: Biobanking, consent and control: a survey of Albertans on key research ethics issues. Biopreserv Biobank 2012; 10: 433-438.

18 Master Z, Claudio JO, Rachul C, Wang J, Minden M, Caulfield T: Cancer patient perceptions on biobanking research. BMC Med Genomics 2013; 6: 8.

19 Heaney C, Tindall G, Lucas J, Haga SB: Researcher practices on returning genetic research results. Genet Test Mol Biomarkers 2010; 14: 821-827.

20 Richer J, Ghebremichael MS, Chudley AE, Robinson WM, Wilfond BS, Solomon MZ: Research use of leftover newborn bloodspots: attitudes of Canadian geneticists regarding storage and informed consent requirements. Genet Med 2011; 13 305-313.

21 Edwards KL, Lemke AA, Trinidad SB et al: Attitudes toward genetic research review: results from a survey of human genetics researchers. Public Health Genomics 2011; 14: 337-345.

22 Massett HA, Atkinson NL, Weber D et al: Assessing the need for a standardized cancer HUman Biobank (caHUB): findings from a national survey with cancer researchers. J Natl Cancer Inst Monogr 2011; 42: 8-15.

23 Meulenkamp TM, Gevers SJK, Bovenberg JA, Smets EMA: Researchers' opinions towards the communication of results of biobank research: a survey study. Eur J Hum Genet 2012; 20: 258-262.
24 Salvaterra E, Giorda R, Bassi MT et al: Pediatric biobanking: a pilot qualitative survey of practices, rules, and researcher opinions in ten European countries. Biopreserv Biobank 2012; 10: 29-36.

25 Garrison NA, Cho MK: Awareness and acceptable practices: IRB and researcher reflections on the Havasupai lawsuit. AJOB Prim Res 2013; 4: 55-63.

26 Ruiz-Canela M, Valle-Mansilla JI, Sulmasy DP: Researchers' preferences and attitudes on ethical aspects of genomics research: a comparative study between the USA and Spain. J Med Ethics 2009; 35: 251-257.

27 Whitley EA, Kanellopoulou N, Kaye J: Consent and research governance in biobanks: evidence from focus groups with medical researchers. Public Health Genomics 2012; 15: 232-242.

28 Master Z, Resnik DB: Incorporating exclusion clauses in informed consent for biobanking. Camb Q Healthc Ethics 2013; 22: 203-212.

29 Strauss A, Corbin J: Basics of Qualitative Research: Grounded Theory Procedures and Techniques. Sage Publications Ltd: Newbury Park, CA, 1990.

30 Charmaz K: Constructing grounded theory. A practical guide through qualitative analysis. Sage Publications Ltd: London, 2006.

31 Hansson MG: Ethics and biobanks. Br J Cancer 2009; 100: 8-12.

32 Otlowski M: Developing an appropriate consent model for biobanks: in defence of 'broad' consent; in Kaye J, Stranger M (eds) Principles and Practice in Biobank Governance. Surrey, England: Ashgate Publishing Limited, 2009; Chapter 5 , pp 79-92.

33 Knoppers BM, Isasi R: Stem cell banking: between traceability and identifiability. Genome Med 2010; 2: 73

34 Gaskell G, Gottweis H: Biobanks need publicity. Nature 2011; 471: 159-160.

35 Hempel C, Lomax G, Peckman S: Broad consent in biobanking. Nat Biotechnol 2012; 30: 826.

36 Gaskell G, Gottweis H, Starkbaum J et al: Public and biobanks: pan-European diversity and the challenge of responsible innovation. Eur J Hum Genet 2013; 21: 14-20.

37 Godard B, Ozdemir V, Fortin M, Egalité N: Ethnocultural community leaders' views and perceptions on biobanks and population specific genomic research: a qualitative research study. Public Underst Sci 2010; 19: 469-485.

38 Lemke A, Wolf WA, Hebert-Beirne J, Smith ME: Public and biobank participant attitudes toward genetic research participation and data sharing. Public Health Genomics 2010; 13: 368-377.

39 Trinidad SB, Fullerton SM, Bares JM, Jarvik GP, Larson EB, Burke W: Genomic research and wide data sharing: views of prospective participants. Genet Med 2010 12: 486-495.

40 Fong M, Braun KL, Chang R: Native Hawaiian preferences for informed consent and disclosure of results from research using stored biological specimens. Pac Health Dialog 2004; 11: 154-159.

41 Mello MM, Wolf LE: The Havasupai Indian tribe case-lessons for research involving stored biologic samples. N Engl J Med 2010; 363: 204-207.

Supplementary Information accompanies this paper on European Journal of Human Genetics website (http://www.nature.com/ejhg) 